\title{
The availability and characteristics of patient-focused YouTube videos related to oral hygiene instruction
}

\author{
Robert S.D. Smyth, ${ }^{1}$ Maya Amlani, ${ }^{2}$ Andrew Fulton, ${ }^{3}$ and Mohammad O. Sharif ${ }^{4 *}$
}

${ }^{1}$ Orthodontic Department, Eastman Dental Hospital, 47-49 Huntley Street, London, WC1E 6DG, UK.

${ }^{2}$ Gertrude's Children's Hospital, Dental Department, Muthaiga, Nairobi, Kenya

${ }^{3}$ Maxillofacial Department, Musgrove park hospital, Taunton

${ }^{4}$ Orthodontic Department, UCL Eastman Dental Institute, 256 Gray's Inn Road, London, WC1X 8LD, UK.

*Correspondence to: Mohammad Owaise Sharif

Email: mohammad.sharif.16@ucl.ac.uk

\section{Key Points}

1. Assesses the availability and quality of the most viewed YouTube videos related to oral hygiene instruction.

2. Highlights important areas that need to be considered when developing patient-focused YouTube videos.

3. Highlights the importance of scientific evidence in informing the content of patient-focused YouTube videos.

\footnotetext{
Abstract

Introduction: YouTube is a popular social media platform that is being increasingly used for the distribution of healthcare related information. To date there appear to be no published studies assessing the quality of oral hygiene instruction provided by YouTube videos.

Objective: To assess the availability, quality and accuracy of patient-focused YouTube videos aiming to provide oral hygiene instruction.

Method: YouTube videos meeting the inclusion criteria were evaluated for quality and information accuracy using an eight-item, evidence-based checklist relating to important aspects in the prevention of caries and periodontal disease.
} 
Results: Fifty-two relevant videos were included, twenty videos (38.5\%) were produced by lay people (social media influencers and bloggers) who had no professional qualifications. None of the included videos contained accurate information relating to all eight items of the evidence-based checklist. Seven videos contained none of the information from the checklist. Numerous videos contained health advice which was not scientifically sound.

Conclusion: The results of this study highlight that currently available YouTube videos may not contain evidence based information relating to oral hygiene instruction. There are also concerns regarding the lack of regulation and quality assurance processes in the development of healthcare related YouTube videos. Currently clinicians should be cautious when advising patients to utilise YouTube as a source of information regarding oral health.

\section{Introduction}

Dental caries and periodontal disease are major healthcare burdens for the United Kingdom (UK) adult and child populations. Whilst there have been significant improvements to the overall oral health of the UK population, obvious dental caries and periodontal disease still affects $31 \%$ and $45 \%$ of the adult population respectively, ${ }^{1}$ while $34 \%$ of children aged 12 years have obvious caries in their permanent dentition. ${ }^{2}$ Preventative interventions have played a key role in reducing caries and periodontal disease levels, ${ }^{3}$ and continue to do so in the UK, as exemplified by initiatives such as the Delivering Better Oral Health: An Evidence-Based Toolkit for Prevention, ${ }^{4}$ the Smile4Life campaign of the Chief Dental Officer, ${ }^{5}$ and the inclusion of prevention in each stage of phased treatment planning. ${ }^{6}$

Preventative healthcare advice is traditionally delivered through verbal and written means, however, this is changing with patients accessing information regarding healthcare from a wide variety of sources, including the internet. ${ }^{7}$ In 2010, 50\% of American adults reported using the internet to supplement their healthcare knowledge in the previous 12 months. ${ }^{8}$ Between 2016 and 2018 , only $50.4 \%$ and $58.6 \%$ of the respective adult and child populations accessed primary dental care. ${ }^{9}$ There is therefore a potential that patients are accessing healthcare information via online resources. In Europe, a 2014 report found $60 \%$ of adults had looked up health information online. ${ }^{10}$ The advent of Web 2.0 has seen the rise of social media platforms and these platforms offer the opportunity for peer to peer interaction as well as social and emotional support. Furthermore, information retrieval may be more effective and personal in comparison to traditional search engines. However, concerns have been raised in regards to the quality and authority of the resources available on such platforms. When patients were asked to rate the trustworthiness of health information sources, while the internet scored $6 / 10$, social 
media scored 3.8/10, with Facebook and Twitter having the lowest scores within the social media bracket. ${ }^{11}$ The potential advantages and disadvantages of social media for patients accessing healthcare information are summarised in Table 1.

YouTube is a popular social media platform that is increasingly being used for the distribution of healthcare-related information. In the UK during 2016, YouTube was the most used social media platform, being accessed by $85 \%$ of the population. ${ }^{12}$ While videos released by government organisations and professional associations may contain high-quality, evidence based and trustworthy information, there is a potential that patients may access videos containing unhelpful and potentially harmful advice. ${ }^{13}$

At present, there are two National Health Service (NHS) YouTube channels: NHS England and NHS Improvement, and NHS. As at October 2019, NHS England and NHS Improvement had 1,306 videos covering a range of topics for both patients and health professionals, including clinical governance, personal experiences, and new technologies being deployed. These videos have been accessed 1,344,207 times and include a subsection for Oral Health Awareness, however, none of the oral health awareness videos have been accessed more than 2,000 times. ${ }^{14}$ These oral health videos predominately cover clinical governance issues relating to dentistry and general videos such as the importance of brushing, but with no detailed instructions regarding oral health care. As at October 2019, the NHS channel had 353 videos which have been accessed 13,700,630 times. This channel has three videos relating to oral health care covering the topics of fluoride (4,100 views), flossing (4,800 views) and how to brush a child's teeth $(14,3000$ views $){ }^{15}$

A review of the existing literature revealed four dental studies relating to the quality of YouTube videos, specifically related to endodontics, oral cancer, dental implants and orthodontics. The evidence relating to endodontic and oral cancer videos has highlighted that these videos vary significantly in terms of quality and completeness of coverage of subject when broken down into categories of aetiology, anatomy, symptoms, procedure, postoperative course and prognosis. ${ }^{16,17}$ Videos relating to dental implants and orthodontics were shown to have low usefulness scores and often misinformed viewers. ${ }^{18,19}$ No studies were found relating to the quality of oral hygiene instruction provided in YouTube videos.

\section{Aims}


The aim of this paper is to assess the availability of patient-focused YouTube videos relating to oral hygiene instruction. Furthermore, the quality and accuracy of frequently accessed YouTube videos relating to oral hygiene instruction is assessed.

\section{Methods}

\section{Search Method}

The following search terms were selected and a YouTube search performed: 'oral hygiene', 'dental hygiene', 'proper brushing', 'tooth brushing', 'teeth cleaning', 'dental flossing', and 'interdental brushing'. The results revealed that some of the search terms were too sensitive and retrieved videos solely providing information on specific aspects of oral hygiene (for example, interdental cleaning). The following search terms were deemed to be most appropriate in retrieving videos pertaining to oral hygiene instruction: 'oral hygiene', 'dental hygiene', 'proper brushing', 'tooth brushing', 'teeth cleaning'. The aforementioned terms were therefore used to search 'http://www.youtube.com' in London, UK. The videos were ordered by number of views, and relevant videos within the top 60 videos retrieved were selected for inclusion in this study. The privacy settings were set so previous searches did not influence new results. Duplicate videos were identified and removed. The time period during which data collection was carried out was three days (13/09/2019-15/09/2019). The videos selected were viewed and assessed by two assessors (MA and AF) for inclusion, the inclusion and exclusion criteria are listed in Table 2. It was intended that a third assessor (MOS) would be consulted to mediate any disagreements relating to inclusion/exclusion of videos.

The generic information extracted from each video included;

1. Year of publication

2. Video duration

3. Number of likes

4. Number of dislikes

5. Number of views

6. Posted by

7. Number of subscribers to the account

The details of who the videos were posted by were further categorised as;

A- Layperson 

B- Healthcare professional
C- Dentist
D- Practice
E- Commercial companies
F- Hospital/University
G- Government body

\section{Quality Assessment}

An 8 item, evidence-based checklist for assessing information relating to the prevention of caries and periodontal disease was used to assess each video for accuracy relating to the information about prevention of caries and periodontal disease. This checklist has previously been reported by Sharif and Alkadhimi ${ }^{20}$ and is presented in Table 3. Each checklist item was scored using the following four-point scale:

1. A: information present, accurate

2. B: information present, incomplete (that is, no inaccurate information but the information present is incomplete, for example, stating that fluoride toothpaste should be used, however failing to provide the recommended fluoride concentration)

3. C: information present, not accurate

4. D: information not present.

\section{Results}

The search identified a total of 300 YouTube videos and after viewing all videos, removing duplicates and cross referencing against the criteria in Table 2, 52 videos were considered eligible for inclusion. The majority of excluded videos were associated with dental hygiene and therapy as a profession, those demonstrating periodontal treatment being carried out and those aimed at pre-school children. Table 4 summarises the characteristics of included videos. All included videos were published between May 2007 and April 2019. The majority of videos were produced by laypeople $(\mathrm{n}=20)$ and there were no videos produced by a Hospital/University. Figure 1 demonstrates the number of likes and dislikes per video, this demonstrates that there are consistently more likes than dislikes for the included videos. Figure 2 demonstrates the number of views per YouTube video when assessed by the role of the person who uploaded the video. The YouTube video with the highest number of views was posted by a dental practice and had 7,724,704 views. Table's 5 and 6 summarise the information content of included video in comparison to the evidence based criteria in Table 3. Item 2 (brushing 
frequency, brush twice a day) had the highest number of videos with information present and accurate (22/52) and item 4 (fluoride content, Use pea-size amount (smear of toothpaste for children up to three years of age) of fluoridated toothpaste (1,350-1,500 ppm fluoride)) had the highest number of videos with information absent (42/52).

Eight of the 52 videos viewed gave incorrect advice on using mouthwash. Seven of the included videos advised rinsing with water after brushing, only one video gave the correct concentration of fluoride to use whilst nine other videos discussed the fluoride content of toothpaste, however, the information provided was incorrect or incomplete. The remainder of the videos made no mention of fluoride content at all, this may be understandable given the potential individual variables that may influence fluoride doses, however, referral for/to professional advice in regards to fluoride content was almost universally absent. Only 6/52 videos contained correct information relating to all aspects of toothbrushing (Items 1-5).

Seventeen of the 52 videos did not mention brushing frequency or duration, which means potentially viewers may be missing out on this advice and may fail to carry out effective oral hygiene measures. With regards to spitting not rinsing after brushing, five videos gave accurate advice, yet a further 39 videos did not mention spitting or rinsing at all. Eighteen videos failed to provide information relating to interdental cleaning and 30 videos gave advice that was incomplete or incorrect.

Figure 3 demonstrates that the YouTube videos with the most views do not routinely correlate with those videos scoring the highest with regards to the evidence-based checklist. Points were allocated based on scores achieved by the videos $(A=4, B=3, C=2, D=1)$, and then correlated against the number of views for each video. Therefore, the maximum score that could be achieved was 32 , which no video attained. The mean score was 15 , with the highest score achieved being 27 . The lowest score recorded was 8 , which 7 of the videos scored.

The year with most publications was $2013(\mathrm{n}=8)$, as at October 2019 only two published videos were available for inclusion in this study and the number of published videos appears to be decreasing year on year since 2016 .

\section{Discussion}

In the digital age, it appears that patients are increasingly drawn to the internet to better understand their medical conditions and treatments, and to help them make informed decisions relating to their care. Fifty-two relevant videos were identified for inclusion in this study, twenty were produced by lay people (social media influencers and bloggers) who appeared to have no professional dental qualifications. Fourteen were produced by dentists or dental 
practices and seven were produced by dental hygienists. One video was produced by an international Dental Association and none of the videos were produced by a university or educational institution. The videos associated with dental practices were often linked to specific treatments they offer. As highlighted in a recent systematic review on healthcare information available on YouTube, it is common that YouTube videos may contain misleading information, primarily anecdotal. This information can be contradictory to the reference standards and the probability of a member of the general public finding such content is actually relatively high. ${ }^{13}$ Interestingly none of the included videos contained accurate information relating to all eight items of the evidence-based checklist for the prevention of caries and periodontal disease. Seven videos contained none of the information listed on the checklist. Where a YouTube account contained more than one video including those that were not focused on oral health it was found that the oral health care videos had significantly fewer views than other non-dentally focused videos. For example, one YouTube blogger received 1.2 million views for a video on make-up, however the oral hygiene video posted by this person had only 9,800 . The highest viewed video identified in this study had 7,724,704 views, this is lower than the highest viewed video in a similar study assessing orthodontic YouTube videos which had a top hit of 40,510,079 views for the search term 'orthodontic treatment' ${ }^{19}$

An overwhelming number of videos did not contain all of the information detailed on the evidence-based checklist for prevention of caries and periodontal disease. This compares to previous research on smartphone apps where the majority of the apps $(n=13)$ did not contain information relating to at least $50 \%$ or more of the checklist items. ${ }^{20}$

Arguably more concerningly, there were videos that contained incorrect/inaccurate/potentially harmful information, some examples of inappropriate advice provided to patients include:

1. Rinsing with water after brushing

2. Rinsing with Coca-Cola

3. Rinsing with hydrogen peroxide

\section{Assessing the quality of YouTube videos:}

There are a number of tools available to help the general public quality assess various forms of digital information, including websites and healthcare apps. ${ }^{21}$ For websites discussing treatment options for various medical procedures, there are two readily available resources, DISCERN and Health on the Net (HON). ${ }^{21}$ When evaluating medical apps, the tool Mobile App Rating Scale (MARS) exists (amongst others). ${ }^{20}$ When evaluating quality assessment tools specific to YouTube videos, it was found that no standardised tool was widely available or 
recognised. ${ }^{21}$ The most common methods of quality assessment utilised by healthcare professionals as part of research were a modified DISCERN / HON tool, creation of a novel scoring system using an evidence-based checklist or subjective categorisation of videos based on knowledge of assessors. ${ }^{21}$ Whilst no standardised tool exists to evaluating the quality of content for individual YouTube videos, we could extrapolate the perceived quality of each video based on the number of likes and dislikes of YouTube using Figure 1. The video which received the highest number of likes scored category D (information not present) in half of the checklist criteria and category $\mathrm{C}$ (information present, not accurate) in a quarter of the checklist criteria. It has been shown in Figure 3 that the YouTube videos with the most views do not correlate with videos scoring the highest with regards to the evidence-based checklist.

The popularity of YouTube and increase in prevalence of patient usage is being reflected by individual organisations releasing institutional guidelines for their employees when producing educational YouTube videos for patients, for example the Center for Disease Control and Prevention. ${ }^{22}$ These are guidelines created for professionals looking to produce educational videos for the general public which would be affiliated with the specific institutions. No such guidelines exist for the NHS yet. As mentioned earlier, the NHS has two YouTube channels, NHS, which was established in 2007 with 42.3k subscribers, and NHS England and NHS Improvements, which was established in 2013 with $47.2 \mathrm{k}$ subscribers. ${ }^{14,15}$ On the NHS England channel, out of the 1306 videos uploaded, there are four videos purportedly related to oral health, none of these videos were in the top 60 videos for any of our search terms. ${ }^{14}$

\section{Unhelpful and potentially harmful content:}

Several videos included in this study provided advice which may be actively detrimental to oral health. A number of videos $(\mathrm{n}=2)$ provided recipes for home-made toothpaste, using mainly tomatoes and bicarbonate of soda. The safety of dentifrices containing bicarbonate of soda has been extensively studied, ${ }^{23-25}$ however these studies do not relate to home-made recipes where the composition is controlled not by a manufacturer but by the person making the toothpaste.

A video identified in the original search but excluded from our analysis (because it related to home tooth whitening tips), recommended the use of household hydrogen peroxide, which is essentially a laundry bleach, for home teeth whitening tips and had over 7 million views. A further excluded video, stated that using toothpaste for sensitive teeth when you are not suffering from sensitivity can cause sensitivity. 


\section{Study limitations}

It is important to highlight that the checklist utilised for assessing knowledge content within the YouTube videos was focused on oral hygiene information provision in the UK. Some of the YouTube videos identified may not have been targeted at a UK patient base, however they were still accessible from within the UK and so there is a need for dental professionals to be aware of the content that patients may be exposed to in order to provide appropriate guidance in relation to online information resources.

Additionally, this research only provides a snapshot of the available YouTube videos relating to oral hygiene instruction in late 2019. The number of available videos is constantly increasing year on year and as such it is sensible to assess the availability and quality of YouTube videos as and when recommending these to patients. Whilst overall the number of YouTube videos is increasing, it was noted in this study that oral hygiene instruction videos was decreasing. This may be due to the rise in popularity of other social media platforms, such as Instagram and Twitter, which people may be using more frequently to upload advice and videos.

\section{Implications for practice and further research:}

A large proportion of the videos viewed by the authors $(n=20)$ were produced by lay people (social media influencers and bloggers) who had no professional qualifications, 14 were produced by dentists or dental practices and seven were produced by dental hygienists (Figure 2). One video was produced by an international Dental Association and no videos were produced by a university or educational institution. The videos associated with dental practices were often linked to specific treatments they offer.

Given the findings of this research, it is essential that the dental profession engage with the public to actively develop high quality evidence-based knowledge for patients in a format that is contemporary and likely to be accessed. At present, dental professionals are advised to assess any YouTube videos they are considering to recommend to their patients for quality and accuracy of information content prior to recommending these to patients.

Furthermore, as recognised in previous research, there is a need to design assessment tools to enable consumers to critically evaluate the videos posted on YouTube with more authoritative information sources to make effective healthcare decisions. ${ }^{13}$ It would also be beneficial to identify effective ways for disseminating trustworthy information on the Internet, be that on YouTube or other platforms, so that it becomes an effective part of our patients healthcare decision-making process. 


\section{Conclusion}

The results of this study highlight that currently available YouTube videos may not contain evidence-based information relating to oral hygiene instruction. There are also concerns regarding the lack of regulation and quality assurance processes in the development of healthcare related YouTube videos. Given the findings of this study, dental professionals are advised to assess any YouTube videos they are considering to recommend to their patients for quality and accuracy of information content prior to recommending these to patients.

\section{Author Contributions}

Mohammad O. Sharif conceived the idea for this project, supervised the project and drafted the manuscript. Maya Amlani and Robert S.D. Smyth contributed equally as co-first authors. Andrew Fulton contributed to data collection and initial drafting of the manuscript.

\section{References}

1. Information Centre for Health and Social Care OfNS, Survey Division. Adult Dental Health Survey, 2009. 2nd Edition: UK Data Service; 2012.

2. Office for National Statistics. Children's Dental Health Survey: 2013. UK Data Service; 2015. Available at: https://digital.nhs.uk/data-andinformation/publications/statistical/children-s-dental-health-survey/child-dental-healthsurvey-2013-england-wales-and-northern-ireland. Accessed 15/09/2019.

3. Garcia RI, Sohn W. The paradigm shift to prevention and its relationship to dental education. J Dent Educ. 2012;76(1):36-45.

4. Public Health England. Delivering Better Oral Health: An Evidence-Based Toolkit for Prevention. 3rd Edition. London: PHE; 2017. Available at:

https://assets.publishing.service.gov.uk/government/uploads/system/uploads/attachment_data /605266/Delivering_better_oral_health.pdf. Accessed: 15/09/2019.

5. NHS England. Smile 4 Life. 2017. Available at: https://www.england.nhs.uk/primary-care/dentistry/smile4life/. Accessed: 15/09/2019. 
6. Office of Chief Dental Officer England. Avoidance of doubt: Provision of phased treatments. 2018. Available at: https://www.england.nhs.uk/wpcontent/uploads/2018/02/avoidance-of-doubt-provision-of-phased-treatments-v20.pdf. Accessed: 15/09/2019.

7. Ramsey I, Corsini N, Peters MDJ, Eckert M. A rapid review of consumer health information needs and preferences. Patient Educ Couns. 2017;100(9):1634-42.

8. Center for Studying Health System Change. Health tracking household survey. 2010. Available at: https://doi.org/10.3886/ICPSR34141.v1. Accessed: 15/09/2019.

9. NHS England. NHS dental statistics for England second quarterly report 2018-19. 2019. Available at: https://digital.nhs.uk/data-and-information/publications/statistical/nhsdental-statistics/quarter-2-2018-19. Accessed: 15/09/2019.

10. European Commission. Flash Eurobarometer 404: European citizens' digital health literacy $\quad$ survey. $2014 . \quad$ Available at: http://ec.europa.eu/commfrontoffice/publicopinion/flash/fl404_en.pdf.

Accessed: 15/09/2019.

11. Zhao Y, Zhang J. Consumer health information seeking in social media: a literature review. Health Info Libr J. 2017;34(4):268-83.

12. Migiro G. Which countries watch the most YouTube? 2018. Available at: http://www.worldatlas.com/articles/which-countries-watch-the-most-youtube.html. Accessed: 15/09/2019.

13. Madathil KC, Rivera-Rodriguez AJ, Greenstein JS, Gramopadhye AK. Healthcare information on YouTube: A systematic review. Health Informatics J. 2015;21(3):173-94.

14. NHS England and NHS Improvement. NHS England and NHS Improvement YouTube channel. 2019. Available at: https://www.youtube.com/user/TheNHSEngland. Accessed: 15/09/2019. 
15. NHS. NHS YouTube channel. 2019. Available at: https://www.youtube.com/user/NHSChoices. Accessed: 19/09/2019.

16. Nason K, Donnelly A, Duncan HF. YouTube as a patient-information source for root canal treatment. Int Endod J. 2016;49(12):1194-200.

17. Hassona Y, Taimeh D, Marahleh A, Scully C. YouTube as a source of information on mouth (oral) cancer. Oral Dis. 2016;22(3):202-8.

18. Abukaraky A, Hamdan AA, Ameera MN, Nasief M, Hassona Y. Quality of YouTube TM videos on dental implants. Med Oral Patol Oral Cir Bucal. 2018;23(4):e463-e8.

19. Kilinc DD, Sayar G. Assessment of Reliability of YouTube Videos on Orthodontics. Turk J Orthod. 2019;32(3):145-50.

20. Sharif MO, Alkadhimi A. Patient focused oral hygiene apps: an assessment of quality (using MARS) and knowledge content. Br Dent J. 2019;227(5):383-6.

21. Drozd B, Couvillon E, Suarez A. Medical YouTube Videos and Methods of Evaluation: Literature Review. JMIR Med Educ. 2018;4(1):e3.

22. Center of Disease Control and Prevention. Social media guidelines and best practices CDC YouTube channel. 2012. Available at: https://www.cdc.gov/SocialMedia/Tools/guidelines/pdf/onlinevideo.pdf. Accessed: 15/09/2019.

23. Myneni SR. Effect of baking soda in dentifrices on plaque removal. J Am Dent Assoc. 2017;148(11S):S4-S9.

24. Li Y. Stain removal and whitening by baking soda dentifrice: A review of literature. $J$ Am Dent Assoc. 2017;148(11S):S20-S6.

25. Ciancio SG. Baking soda dentifrices and oral health. $J$ Am Dent Assoc. 2017;148(11S):S1-S3. 
Table 1. Advantages and disadvantages of social media use in accessing healthcare information

\begin{tabular}{|l|l|}
\hline Advantages & Disadvantages \\
\hline Peer-to-peer information sharing and & Non-credible sources of information \\
\hline discussion & Outdated information \\
\hline Personalised information retrieval & Non-scientific content (potentially harmful) \\
\hline Empowering/engaging & \\
\hline Easily accessible/free & \\
\hline
\end{tabular}

Table 2. Inclusion and exclusion criteria

\begin{tabular}{|l|l|}
\hline Inclusion Criteria & Exclusion Criteria \\
\hline $\begin{array}{l}\text { Videos aimed at providing oral hygiene } \\
\text { instruction }\end{array}$ & $\begin{array}{l}\text { Videos aimed at dental professionals or } \\
\text { undergraduate students }\end{array}$ \\
\hline Videos aimed at promoting oral health & Non-English language videos \\
\hline Videos aimed at treating bad breath & $\begin{array}{l}\text { Videos with background music audio } \\
\text { only }\end{array}$ \\
\hline $\begin{array}{l}\text { Videos aimed at providing oral health } \\
\text { instruction and bleaching }\end{array}$ & $\begin{array}{l}\text { Videos specifically focused on flossing or } \\
\text { interdental brushing }\end{array}$ \\
\hline & \begin{tabular}{l} 
Videos specifically focused on bleaching \\
\hline
\end{tabular} \\
\hline & $\begin{array}{l}\text { Videos aimed at pre-school children } \\
\text { appliances }\end{array}$ \\
\hline & Videos not related to oral hygiene \\
\hline
\end{tabular}

Table 3. An evidence-based checklist for assessing information relating to the prevention of caries and periodontal disease

\begin{tabular}{|l|l|}
\hline Code & Item \\
\hline Item 1 & Brush teeth for at least two minutes with fluoridated toothpaste \\
\hline Item 2 & Brush at least twice a day \\
\hline Item 3 & Brush last thing at night and at least on one other occasion \\
\hline
\end{tabular}




\begin{tabular}{|l|l|}
\hline Item 4 & $\begin{array}{l}\text { Use pea-size amount (smear of toothpaste for children up to three years of } \\
\text { age) of fluoridated toothpaste (1,350-1,500 ppm fluoride) }\end{array}$ \\
\hline Item 5 & $\begin{array}{l}\text { Spit out after brushing and do not rinse to maintain fluoride concentration } \\
\text { levels }\end{array}$ \\
\hline Item 6 & $\begin{array}{l}\text { Use fluoride containing mouth rinse daily (0.05\% NaF) at a different time to } \\
\text { brushing (indicated in high caries risk individuals) }\end{array}$ \\
\hline Item 7 & $\begin{array}{l}\text { Small toothbrush head of medium texture } \\
\text { interdental or single tufted brushes }\end{array}$ \\
\hline Item 8 & \\
\hline
\end{tabular}


Table 4. Characteristics of included YouTube videos assessed. Posted by A=Layperson, B=Healthcare professional, C=Dentist, $D=$ Practice, E=Commerical companies, F=Hospital/University, G=Government body.

\begin{tabular}{|c|c|c|c|c|c|c|c|}
\hline $\begin{array}{l}\text { Video } \\
\text { number }\end{array}$ & $\begin{array}{l}\text { Year of } \\
\text { publication }\end{array}$ & $\begin{array}{l}\text { Duration in } \\
\text { minutes and } \\
\text { seconds }\end{array}$ & Likes & Dislikes & $\begin{array}{l}\text { Number } \\
\text { of views }\end{array}$ & $\begin{array}{l}\text { Posted } \\
\text { by }\end{array}$ & $\begin{array}{l}\text { Number of } \\
\text { subscribers } \\
\text { to account }\end{array}$ \\
\hline 1 & 2011 & 12.13 & 3777 & 146 & 543556 & $B$ & 1320 \\
\hline 2 & 2007 & 06.58 & 3700 & 155 & 409399 & B & 22500 \\
\hline 3 & 2013 & $04: 54$ & 846 & 126 & 267332 & $B$ & not visible \\
\hline 4 & 2015 & $02: 33$ & 1741 & 133 & 250296 & $\mathrm{C}$ & 659247 \\
\hline 5 & 2012 & $04: 26$ & 1131 & 117 & 234128 & $A$ & 43090 \\
\hline 6 & 2012 & $02: 11$ & not visible & not visible & 167492 & $E$ & 185 \\
\hline 7 & 2013 & $07: 43$ & 2728 & 95 & 162452 & $A$ & 464111 \\
\hline 8 & 2016 & $01: 37$ & 308 & 23 & 142523 & $A$ & 241719 \\
\hline 9 & 2016 & 10.50 & 9800 & 189 & 380961 & $A$ & 624 \\
\hline 10 & 2019 & 10.32 & 4200 & 64 & 198004 & $A$ & 163000 \\
\hline 11 & 2013 & 07.43 & 2700 & 96 & 165085 & $A$ & 468000 \\
\hline 12 & 2016 & 05.00 & 4400 & 134 & 121617 & $A$ & 1090000 \\
\hline 13 & 2019 & 13.12 & 0 & 0 & 115161 & $A$ & 241000 \\
\hline 14 & 2013 & 14.14 & 24000 & 2500 & 3466217 & $A$ & 2200000 \\
\hline 15 & 2018 & 00.20 & 18 & 11 & 2288257 & $E$ & 0 \\
\hline 16 & 2017 & 12.50 & 1600 & 609 & 1004955 & $E$ & 28000000 \\
\hline 17 & 2016 & 04.18 & 1700 & 136 & 776529 & $E$ & 276000 \\
\hline 18 & 2007 & 02.38 & 1000 & 90 & 730203 & $B$ & 3500000 \\
\hline 19 & 2018 & 07.27 & 4300 & 239 & 295951 & A & 3530000 \\
\hline 20 & 2016 & 07.20 & 5900 & 148 & 261666 & $A$ & 5340000 \\
\hline 21 & 2013 & 01.29 & 636 & 41 & 167189 & $D$ & 4210 \\
\hline 22 & 2015 & 00.14 & 2 & 2 & 149777 & $E$ & 4260 \\
\hline 23 & 2016 & 01.37 & 321 & 24 & 145772 & $E$ & 243000 \\
\hline 24 & 2015 & 05.10 & 5200 & 47 & 136626 & $A$ & 527000 \\
\hline
\end{tabular}




\begin{tabular}{|c|c|c|c|c|c|c|c|}
\hline 25 & 2015 & 03.08 & 10000 & 356 & 2171912 & $C$ & 2760000 \\
\hline 26 & 2008 & 01.49 & 899 & 138 & 424487 & $A$ & 24687 \\
\hline 27 & 2018 & 10.16 & 22637 & 2595 & 2700951 & $A$ & 59564072 \\
\hline 28 & 2017 & 03.21 & 2796 & 1153 & 2659712 & $A$ & 143936 \\
\hline 29 & 2017 & 08.19 & 10400 & 434 & 592693 & $A$ & 4190 \\
\hline 30 & 2013 & 01.56 & 3100 & 186 & 525108 & $\mathrm{D}$ & 524 \\
\hline 31 & 2013 & 09.18 & 649 & 49 & 91915 & $A$ & 125000 \\
\hline 32 & 2012 & 03.29 & 8108 & 1440 & 7724704 & $\mathrm{D}$ & 39500 \\
\hline 33 & 2011 & 04.53 & 8120 & 1317 & 3079162 & $E$ & 11109 \\
\hline 34 & 2017 & 04.34 & 13756 & 1543 & 2956676 & $A$ & 44595 \\
\hline 35 & 2011 & 01.20 & 4516 & 831 & 2142126 & $E$ & 3058699 \\
\hline 36 & 2015 & 9.0 & 25532 & 1735 & 2065313 & $A$ & 3087 \\
\hline 37 & 2015 & 6.10 & 6228 & 546 & 1197922 & $C$ & 2382851 \\
\hline 38 & 2012 & 4.09 & 3103 & 289 & 831231 & $A$ & 3726 \\
\hline 39 & 2007 & 2.38 & 1047 & 90 & 730193 & B & 674126 \\
\hline 40 & 2009 & 1.35 & 1000 & 101 & 603829 & $C$ & 3532929 \\
\hline 41 & 2010 & 2.00 & 1500 & 146 & 421542 & $D$ & 1910 \\
\hline 42 & 2014 & 0.59 & not visible & not visible & 325021 & $E$ & 888 \\
\hline 43 & 2014 & 1.00 & 574 & 74 & 320445 & G & 15600 \\
\hline 44 & 2014 & 2.22 & 1500 & 57 & 318983 & $C$ & 7160 \\
\hline 45 & 2013 & 6.26 & 892 & 82 & 209945 & $C$ & 3870 \\
\hline 46 & 2010 & 2.35 & 362 & 29 & 181317 & $C$ & 539 \\
\hline 47 & 2015 & 1.51 & 319 & 84 & 139954 & $B$ & 16500 \\
\hline 48 & 2014 & 2.43 & 529 & 69 & 127689 & $\mathrm{D}$ & 247 \\
\hline 49 & 2007 & 3.09 & 165 & 22 & 120064 & $C$ & 3500000 \\
\hline 50 & 2014 & 8.57 & 445 & 56 & 119680 & $B$ & 26800 \\
\hline 51 & 2016 & 2.56 & 272 & 72 & 111888 & $E$ & 194 \\
\hline 52 & 2012 & 2.32 & 642 & 23 & 98066 & $C$ & 7160 \\
\hline
\end{tabular}


Table 5. The knowledge content of YouTube videos included. Each criteria of the evidence-based checklist was scored as follows. A: information present, accurate; B: information present, incomplete; $C$ : information present, not accurate; and $D$ : information not present 


\begin{tabular}{|c|c|c|c|c|c|c|c|c|c|}
\hline $\begin{array}{l}\text { Video } \\
\text { Number }\end{array}$ & $\begin{array}{l}\text { Number } \\
\text { of views }\end{array}$ & $\begin{array}{l}\text { 1.Brushing } \\
\text { duration }\end{array}$ & $\begin{array}{l}\text { 2.Brushing } \\
\text { frequency }\end{array}$ & $\begin{array}{l}\text { 3.Brushing } \\
\text { time }\end{array}$ & $\begin{array}{l}\text { 4.Fluoride } \\
\text { content }\end{array}$ & 5.Rinsing & 6.Mouthrinse & $\begin{array}{l}\text { 7.Toothbrush } \\
\text { size and } \\
\text { hardness }\end{array}$ & $\begin{array}{l}\text { 8.Interdental } \\
\text { Cleaning }\end{array}$ \\
\hline 1 & 543556 & A & A & $\mathrm{D}$ & $\mathrm{D}$ & $\mathrm{D}$ & $\mathrm{D}$ & $B$ & B \\
\hline 2 & 409399 & $D$ & B & B & D & D & C & B & B \\
\hline 3 & 267332 & D & D & D & D & D & C & B & B \\
\hline 4 & 250296 & A & A & A & C & $\mathrm{D}$ & B & B & B \\
\hline 5 & 234128 & A & A & A & A & A & D & B & B \\
\hline 6 & 167492 & D & A & B & D & D & B & D & B \\
\hline 7 & 162452 & $\mathrm{D}$ & A & $\mathrm{D}$ & B & $C$ & $\mathrm{C}$ & $\mathrm{D}$ & B \\
\hline 8 & 142523 & $D$ & A & $\mathrm{D}$ & $\mathrm{D}$ & $\mathrm{C}$ & $B$ & $\mathrm{D}$ & B \\
\hline 9 & 380961 & D & A & D & D & D & B & B & B \\
\hline 10 & 198004 & D & D & D & B & A & B & D & D \\
\hline 11 & 165085 & D & D & D & D & D & C & D & B \\
\hline 12 & 121617 & D & C & D & D & D & D & D & B \\
\hline 13 & 115161 & D & C & D & D & C & C & D & C \\
\hline 14 & 3466217 & D & A & B & D & D & D & C & B \\
\hline 15 & 2288257 & D & D & D & D & D & B & D & D \\
\hline 16 & 1004955 & D & A & D & D & D & B & D & B \\
\hline 17 & 776529 & $\mathrm{D}$ & A & B & $\mathrm{D}$ & $\mathrm{D}$ & $\mathrm{D}$ & $\mathrm{D}$ & $\mathrm{D}$ \\
\hline 18 & 730203 & A & A & C & D & D & D & D & D \\
\hline 19 & 295951 & D & D & D & D & D & $\mathrm{D}$ & D & $\mathrm{D}$ \\
\hline 20 & 261666 & A & A & A & D & D & D & D & $B$ \\
\hline 21 & 167189 & A & A & A & D & D & D & D & B \\
\hline 22 & 149777 & B & B & B & D & D & D & D & B \\
\hline 23 & 145772 & D & D & D & D & C & B & D & B \\
\hline 24 & 136626 & D & D & A & D & D & D & B & B \\
\hline 25 & 2171912 & D & A & B & D & D & D & D & B \\
\hline 26 & 424487 & A & A & $\mathrm{D}$ & B & C & C & $B$ & $\mathrm{D}$ \\
\hline 27 & 2700951 & A & B & D & D & D & D & D & D \\
\hline 28 & 2659712 & D & D & D & D & D & D & D & B \\
\hline 29 & 592693 & C & D & D & D & A & A & $B$ & B \\
\hline 30 & 525108 & D & A & D & B & C & B & $\mathrm{D}$ & B \\
\hline 31 & 91915 & D & D & D & D & D & C & D & B \\
\hline
\end{tabular}




\begin{tabular}{|c|c|c|c|c|c|c|c|c|c|}
\hline 32 & 7724704 & A & A & A & C & A & D & A & A \\
\hline 33 & 3079162 & D & D & D & D & D & D & $\mathrm{D}$ & B \\
\hline 34 & 2956676 & A & D & D & D & D & D & A & B \\
\hline 35 & 2142126 & D & $D$ & $D$ & D & D & D & D & D \\
\hline 36 & 2065313 & A & $D$ & $D$ & D & B & C & B & C \\
\hline 37 & 1197922 & A & D & D & D & D & D & D & A \\
\hline 38 & 831231 & A & A & C & D & D & D & B & D \\
\hline 39 & 730193 & A & A & A & D & D & D & D & D \\
\hline 40 & 603829 & D & A & A & D & D & D & B & D \\
\hline 41 & 421542 & D & B & $A$ & B & D & D & B & D \\
\hline 42 & 325021 & D & D & D & D & D & D & D & D \\
\hline 43 & 320445 & A & A & B & B & D & D & B & B \\
\hline 44 & 318983 & D & D & D & D & D & D & D & D \\
\hline 45 & 209945 & D & D & D & D & D & D & D & B \\
\hline 46 & 181317 & D & D & $D$ & D & c & D & B & B \\
\hline 47 & 139954 & D & A & A & C & A & D & B & A \\
\hline 48 & 127689 & A & D & D & D & D & D & D & D \\
\hline 49 & 120064 & $\mathrm{D}$ & $D$ & $D$ & D & D & D & D & D \\
\hline 50 & 119680 & A & C & A & D & D & D & D & A \\
\hline 51 & 111888 & D & $D$ & $D$ & $D$ & D & D & D & D \\
\hline 52 & 98066 & D & D & D & D & D & D & D & D \\
\hline
\end{tabular}


Table 6. Analysis of the information content of the 8 item evidence-based checklist

\begin{tabular}{|l|l|l|l|l|}
\hline & $\begin{array}{l}\text { Number of } \\
\text { videos scoring A }\end{array}$ & $\begin{array}{l}\text { Number of } \\
\text { videos scoring B }\end{array}$ & $\begin{array}{l}\text { Number of } \\
\text { videos scoring C }\end{array}$ & $\begin{array}{l}\text { Number of } \\
\text { videos scoring D }\end{array}$ \\
\hline Item 1 & 17 & 1 & 1 & 33 \\
\hline Item 2 & 22 & 4 & 3 & 23 \\
\hline Item 3 & 11 & 7 & 2 & 32 \\
\hline Item 4 & 1 & 6 & 3 & 42 \\
\hline Item 5 & 5 & 1 & 7 & 39 \\
\hline Item 6 & 1 & 9 & 8 & 34 \\
\hline Item 7 & 2 & 16 & 1 & 33 \\
\hline Item 8 & 4 & 28 & 2 & 18 \\
\hline
\end{tabular}

Figure 1. Number of likes and dislikes recorded per YouTube video

Figure 1. Number of likes and dislikes recorded per YouTube video

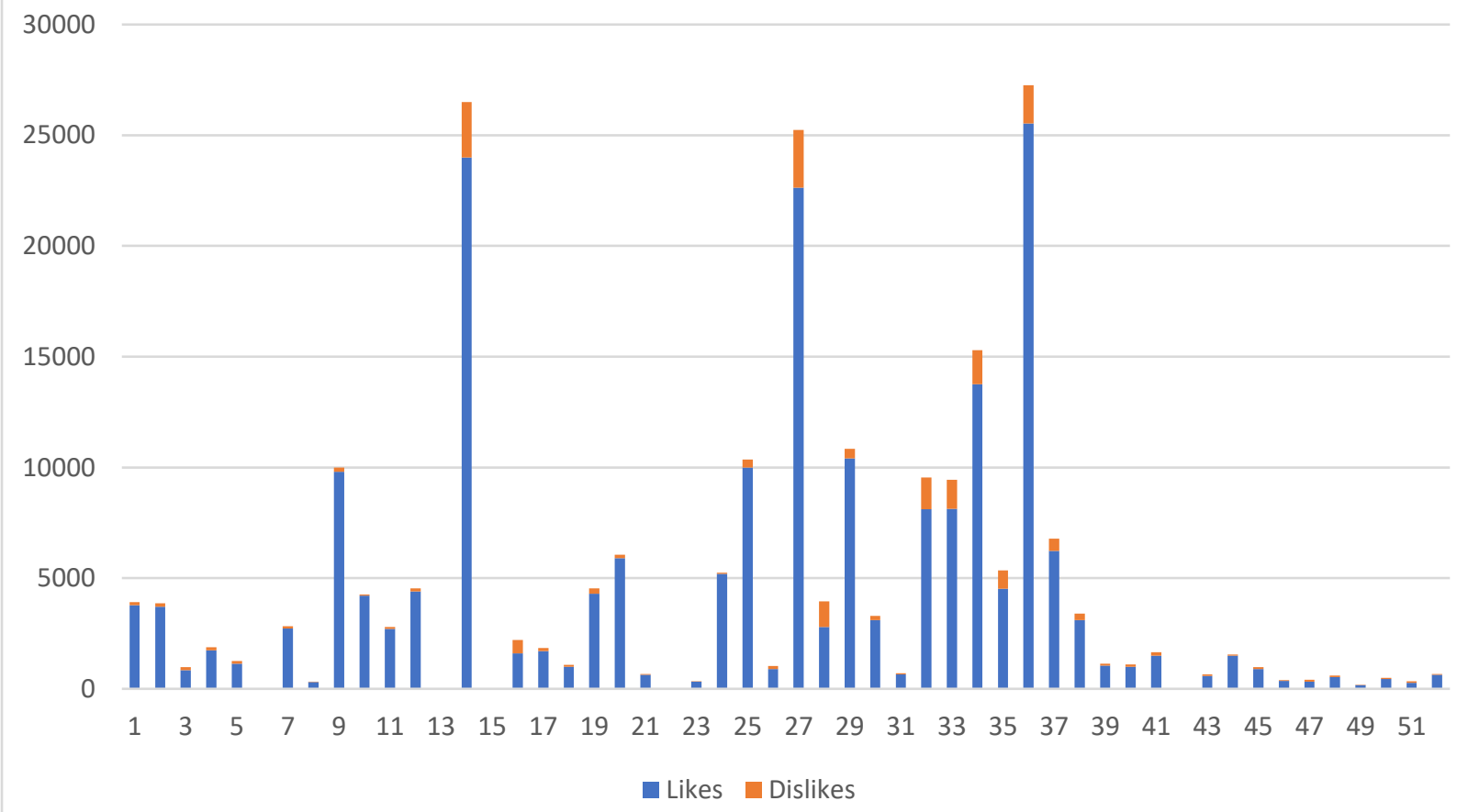


Figure 2. Number of views per YouTube video

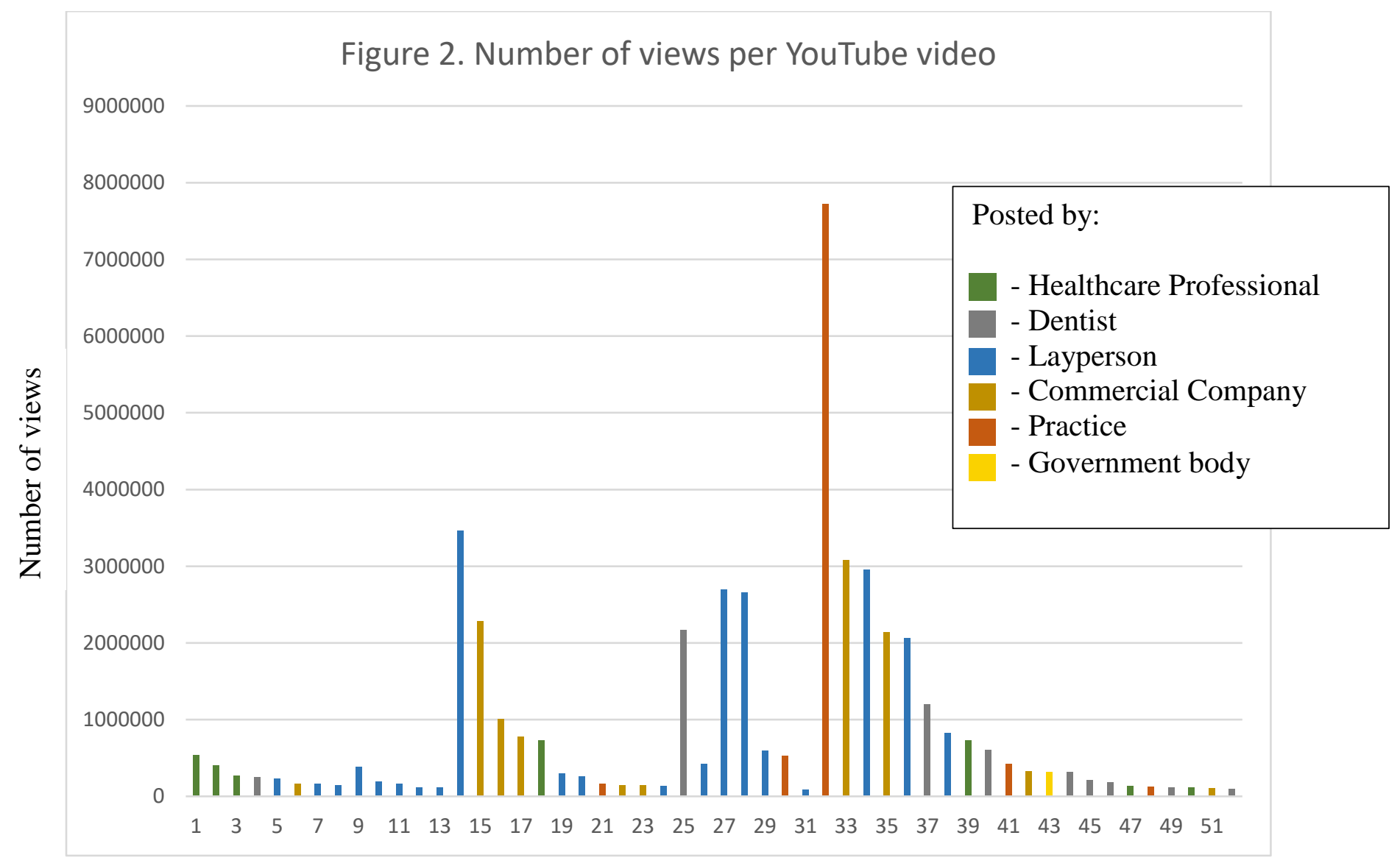

Video Number 
Figure 3. Comparing the accuracy of the YouTube video content with the number of likes per video.

Video Accuracy Vs Number of Views

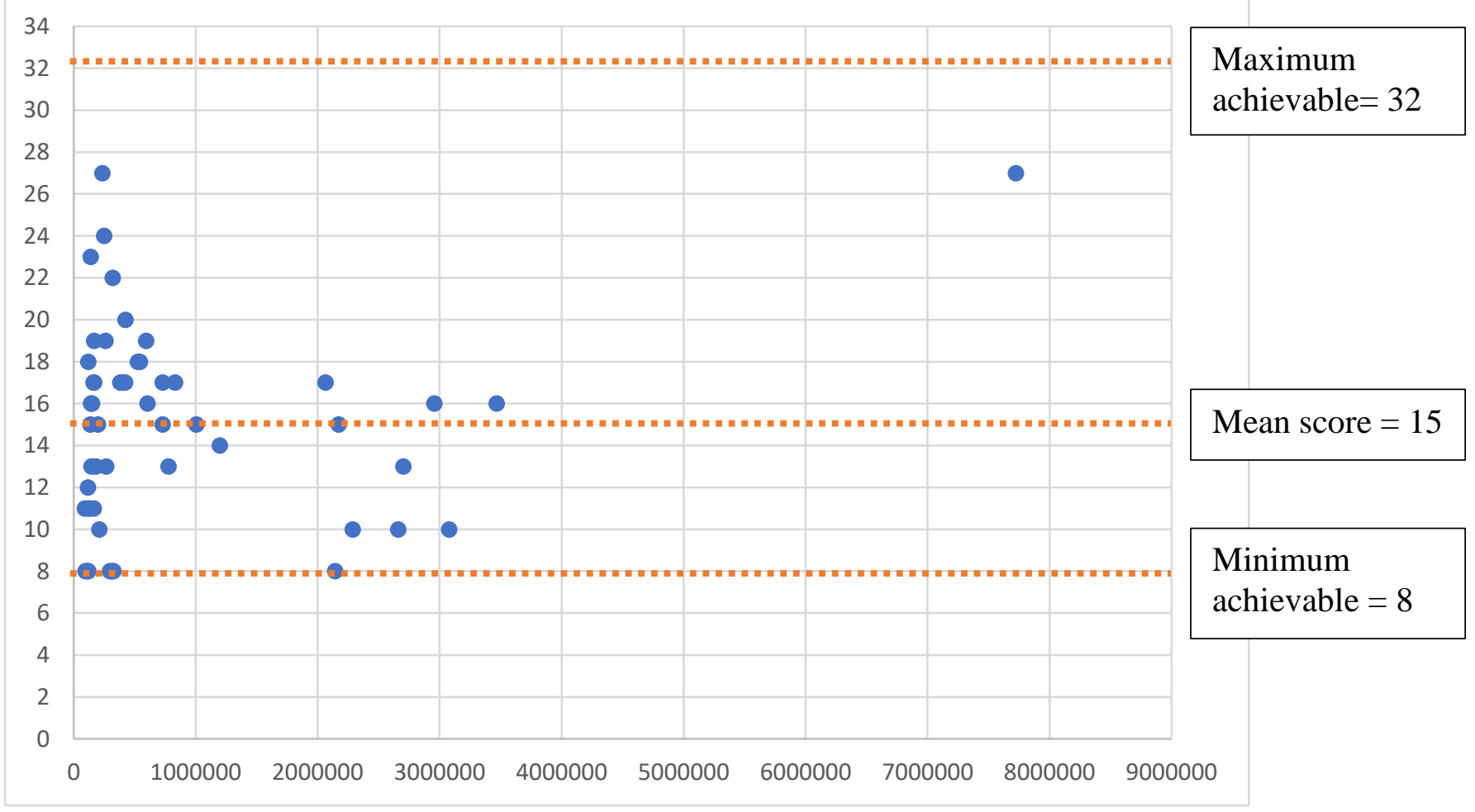

Number of Views 